\title{
ROBINSON'S CONSISTENCY THEOREM IN SOFT MODEL THEORY
}

BY

\author{
DANIELE MUNDICI
}

\begin{abstract}
In a soft model-theoretical context, we investigate the properties of logics satisfying the Robinson consistency theorem; the latter is for many purposes the same as the Craig interpolation theorem together with compactness. Applications are given to H. Friedman's third and fourth problem.
\end{abstract}

Introduction. No extension of first order logic is known which is axiomatizable and/or countably compact, and has some kind of interpolation or definability property such as the Robinson consistency theorem or the Craig interpolation theorem. For the case $L \geqslant L\left(Q_{1}\right)$ (where $Q_{1}$ is the quantifier "there exist uncountably many"), by a result in [Hu] we have, on one hand, failure of Craig interpolation in $\Delta L\left(Q_{1}\right)$ and, on the other hand, in [Mu1] it is proved that no countably compact (resp., no axiomatizable) extension of $L\left(Q_{1}\right)$ will satisfy the Robinson consistency theorem. Both these results seem to support the feeling expressed in [Br] about the nonexistence of countably compact extensions of $L\left(Q_{1}\right)$ satisfying the interpolation property. In the general case when $L \neq L\left(Q_{1}\right)$, less is known.

The Robinson consistency theorem is a very important soft model-theoretical notion; in a somewhat weaker form it was investigated by Makowsky and Shelah in [MS]; in the present form it is studied in [Mu1]-[Mu5] and in [MS1]; in [Mu3], [Mu4] and, independently, in [MS1] such identities are proved as

$$
\text { Robinson Consistency = Compactness + Craig Interpolation, }
$$

and

\section{Compactness $=\mathrm{JEP}$}

(i.e. the Joint Embedding Property of $L$-elementary embeddings).

In this paper we give an exposition of the methods and results about the Robinson consistency theorem in abstract (soft) model theory. We assume familiarity with [Fe2], [Fl], [Ba], [MSS] and with [MS, §6].

In Theorem 3.1 we prove that if in $L$ the Robinson consistency theorem holds, then either $L$ is countably compact, or the theories of $L$ can characterize up to isomorphism all the structures whose cardinality is less than $\omega_{\omega}$. Notice that no special set-theoretical assumption is involved in the proof of this theorem. Corollary 3.3, improving [MS, 6.11], states that if $2^{\omega}<2^{\omega_{n}}$ for some $n \in \omega$, then the

Received by the editors December 1, 1979.

1980 Mathematics Subject Classification. Primary 03C95, 03C40, 03C75, 03C80; Secondary 03C55, 03C30, 03E55. 
Robinson consistency theorem implies countable compactness and the Craig interpolation theorem is any countably generated logic. Notice that this result was obtained by Makowsky and Shelah via an additional "Feferman Vaught" assumption.

In $\$ 4$ we take up extensions of $L\left(Q_{1}\right)$ in the light of [Mu1]; then Theorem 3.1 immediately yields that if $L \geqslant L\left(Q_{1}\right)$ satisfies the Robinson consistency theorem, then $L$ can characterize all structures up to cardinal $\omega_{\omega}$ (by means of their complete theories in $L$ ), and $L$ is a very large logic which is neither axiomatizable nor countably compact. Under (something weaker than) $\mathrm{CH}$ we also prove in Corollaries 4.2 and 4.3 that any such $L$ necessarily has uncountably many quantifiers and that the real numbers can be characterized by a theory in $L$.

In $\S 5$ (under $\neg 0^{\#}$ or $\neg L^{\mu}$ ) we prove that if $\operatorname{Stc}_{L}(\tau)$ is a set for $\tau$ a set, then $L$ satisfies the Robinson consistency theorem iff $L$ is compact and satisfies the Craig interpolation theorem. In [Mu3] this was proved under $\neg 0^{\#}$. The proof is almost the same if one uses $\neg L^{\mu}$, see [Mu4], or even if one assumes that $\forall \kappa$ regular $>\omega$, for any uniform ultrafilter $D$ on $\kappa, \forall \lambda \geqslant \omega, D$ is $\lambda$-descendingly incomplete. From the main theorem in [MS1], the same identity can be established by assuming that there is no measurable cardinal $>\omega$, and that $L$ has an "occurrence number".

In $\$ 6$ we prove some results about $\mathrm{H}$. Friedman's third and fourth problem in [Fr]; namely, in Theorem 6.1, under $\neg 0^{\sharp}$ or $\neg L^{\mu}$, we show that no logic $L$ between $L_{\infty \omega}$ and $L_{\infty \infty}$ satisfies the Craig interpolation (or the Robinson consistency) theorem, unless $\equiv_{L}=\cong$. In Theorems 6.2 and 6.3 we prove two results whose effect is practically that any logic $L$ satisfying the Robinson consistency theorem together with some kind of Löwenheim requirement will automatically have $\equiv_{L}$ equal to $\equiv$ on the class of countable structures of finite type.

In conclusion, we do agree with the observation by Makowsky and Shelah in [MS], that with more effort more theorems about (the interpolation and definability properties in) abstract model theory should be provable.

Still, we wonder why theorems in soft model theory may be so hard.

0. Preliminaries. Ordinals and, in particular, cardinals, are denoted by small Greek letters; the first infinite cardinals are written $\omega=\omega_{0}, \omega_{1}, \ldots, \omega_{\omega}, \ldots$ We shall be working throughout with logics $L$ as defined in [FI] and/or in [Fe2]. Unexplained notation is standard; for $\tau$ a (similarity) type, $\operatorname{Str}(\tau)$ is the class of all structures of type $\tau, \operatorname{Stc}_{L}(\tau)$ is the class of all sentences in logic $L$ of type $\tau$ (following [Fe1] and [Fe2]); notice that what we call a type is called a language by many authors. For $T \subseteq \operatorname{Stc}_{L}(\tau)$ a theory in $L$ of type $\tau, \bmod _{L} T$ is the class of models of $T$; for $\mathfrak{A} \in \operatorname{Str}(\tau), \operatorname{th}_{L} \mathfrak{A}$ is the complete theory of $\mathfrak{A}$ in $L$ of type $\tau$. Following [MSS], we let $I \mathfrak{A}=\{\mathfrak{B} \mid \mathfrak{B} \cong \mathfrak{A}\}$; given $\mathfrak{A}, \mathfrak{B} \in \operatorname{Str}(\tau)$ and logic $L$, $\mathfrak{A} \equiv_{L} \mathfrak{B}$ means that $\operatorname{th}_{L} \mathfrak{A}=\operatorname{th}_{L} \mathfrak{B}$. Notice that $\tau$ may be infinite, and $\operatorname{Stc}_{L}(\tau)$ may be a proper class.

0.1 Definition. Given logic $L$ and structure $\mathfrak{A} \in \operatorname{Str}(\tau)$ we say that in $L, \mathfrak{A}$ is characterizable by its theory in type $\tau$ iff $\bmod _{L} \operatorname{th}_{L} \mathfrak{A}=I \mathfrak{A}$; that is to say that any model $\mathfrak{B}$ of the complete theory of $\mathfrak{A}$ in type $\tau$ is isomorphic to $\mathfrak{A}$; when there is no ambiguity we drop mention of $L$. For $\lambda$ an ordinal, the characterizability of $\lambda$ (or, 
equivalently, of $\langle\lambda,\langle\rangle$ ) by its theory in type $\{<\}$, with $<$ a binary relation symbol, means of course that $\bmod _{L} \operatorname{th}_{L}\left\langle\lambda,\langle\rangle=I\left\langle\lambda,\langle\rangle=I\left\langle\lambda,\left.\varepsilon\right|_{\lambda}\right\rangle\right.\right.$. An important feature of logics $L$ is their closure under relativization of formulas to formulas; for the case $L=L_{\omega \omega}\left(Q^{i}\right)_{i \in I}$, [MSS, p. 159] note that relativization is built in the formation rules for formulas. We shall have to relativize a sentence $\psi$ to formula $\varphi\left(x, y_{1}, \ldots, y_{r}\right)$ with $y_{1}, \ldots, y_{r}$ acting as parameters; we shall then write

$$
\psi^{\left\{x \mid \varphi\left(x, y_{1}, \ldots, y_{r}\right)\right\}} \text {. }
$$

Notice that $[\mathbf{M S}]$ write instead $\psi^{\varphi\left(x, y_{1}, \ldots, y_{r}\right)}$.

If $\mathfrak{A} \in \operatorname{Str}(\tau)$ and $A^{\prime}$ is contained in the universe $A$ of $\mathfrak{A}$ and is nonvoid on each sort of $\tau$, then $\mathfrak{U} \mid A^{\prime}$ denotes the substructure of $\mathfrak{U}$ generated by $A^{\prime}$.

0.2 Definition. Logic $L$ has the Robinson property (or, equivalently, in $L$ the Robinson consistency theorem holds) iff for any theories $T, T_{1}$ and $T_{2}$ and types $\tau, \tau_{1}$ and $\tau_{2}$ with $\tau=\tau_{1} \cap \tau_{2}$, if $T_{1}$ and $T_{2}$ are consistent extensions of $T$ respectively in type $\tau_{1}$ and $\tau_{2}$, and $T$ is complete in $\tau$, then $T_{1} \cup T_{2}$ is consistent in $\tau_{1} \cup \tau_{2}$.

For the definition of other interpolation and definability properties, see [MSS] and [MS].

We recall that logic $L$ is $(\omega, \omega)$-compact (or, equivalently, countably compact) iff each inconsistent countable theory in $L$ has some inconsistent finite subtheory.

\section{From ordinals to arbitrary structures.}

1.1 Proposition. Let logic $L$ have the Robinson property; assume that $L$ is not $(\omega, \omega)$-compact; then $\left\langle\omega,\langle\rangle\right.$ is characterizable in $L$ by its theory $T_{0}=\operatorname{th}_{L}\langle\omega,\langle\rangle$ in type $\{E\}$ with $E$ a binary relation symbol.

Proof. Since $L$ is not countably compact then for some type $\tau^{\prime} \supseteq\{E\}$ and some (countable) consistent theory $T^{\prime}$ of type $\tau^{\prime}$ we have

$$
\forall \mathfrak{U} \in \operatorname{Str}\left(\tau^{\prime}\right), \mathfrak{U}^{\prime} \vDash T^{\prime} \text { implies } \mathfrak{U}^{\prime} \uparrow\{E\} \cong\langle\omega,\langle\rangle
$$

(see [MS, 1.3] who refer to [Ba] and [FI], or see the proof of Theorem 2.1 in this paper). Assume now that $T_{0}$ has some model $\mathfrak{B} \approx\langle\omega,<\rangle$ (absurdum hypothesis). Structure $\mathfrak{B}$ is still a discrete linear ordering with an initial element, and has some expansion $\mathfrak{B}^{\prime}=\langle\mathfrak{B}, P, f, b\rangle$ ( $P$ unary relation, $f$ unary function, $b$ a constant, all symbols not already in $\tau^{\prime}$ ) satisfying the sentence $\psi$ (of $L_{\omega \omega}$ ) which says that

" $E$ is a discrete linear ordering with an initial element and $f$ maps the set $P$ of predecessors of $b$ one-one onto $P \cup\{b\}$ ".

Now $T_{0} \cup\{\psi\}$ is a consistent extension of $T_{0}$ and $T_{0} \cup\{\psi\} \cup T^{\prime}$ is inconsistent, by the above properties of theory $T^{\prime}$. This contradicts the assumed Robinson property of $L$. Q.E.D.

1.2 TheORem. Let $L$ have the Robinson property and $n \in \omega$; assume that each ordinal $\lambda \leqslant \omega_{n}$ is characterizable in $L$ by its theory $T_{\lambda}$ of type $\{E\}$, with $E$ a binary relation symbol. Then for any single-sorted structure $\mathfrak{A}$ such that $|\mathfrak{A}|<\omega_{n}$, we have that $\bmod _{L}$ th $\mathscr{L}=I \mathfrak{A}$. 
REMARK. The single-sortedness assumption is only for simplicity. For the proof we prepare

1.3 LEMMA. Under the hypotheses of Theorem 1.2, we have that for any cardinal $\kappa \leqslant \omega_{n}, \kappa$ is characterizable in $L$ by its theory $T^{\kappa}$ in the pure identity language (viz., in the single-sorted type having no symbols; notice that equality is available in any case).

Proof. Assume (absurdum hypothesis) that some model $M$ of $T^{\kappa}$ with $|M|=\mu$ is such that $\mu \neq \kappa$; then we proceed by cases.

Case one. $\mu>\kappa$. Add $\mu$ many new constants $a_{0}, a_{1}, \ldots$, and extend $T^{\kappa}$ to theory $T^{\prime}$ obtained by adding axioms $a_{\alpha} \neq a_{\beta}$ (whenever $\alpha \neq \beta$ ). Let $T_{\kappa}$ denote $\operatorname{th}_{L}\langle\kappa,<\rangle$ of type $\{E\}$, where $\langle\kappa,<\rangle$ is the least ordinal of cardinality $\kappa$. By our hypothesis about $T_{\kappa}, T^{\prime} \cup T_{\kappa}$ is inconsistent, which contradicts the assumed Robinson property.

Case two. $\mu<\kappa$. Expand $\mu$ to a well order $\left\langle\mu,\langle\rangle\right.$ and let $T_{\mu}$ be its theory in type $\{E\}$. By assumption, all the models of $T_{\mu}$ are isomorphic, hence, in particular, their cardinality is $\mu$. Let $T_{\kappa}^{\prime}$ be the theory in type $\left\{E^{\prime}\right\}$ of the least ordinal $\langle\kappa,\langle\rangle$ of cardinality $\kappa$, with $E^{\prime}$ a new binary relation symbol. By assumption, all the models of $T_{\kappa}^{\prime}$ have cardinality $\kappa$, therefore $T^{\kappa} \cup T_{\mu} \cup T_{\kappa}^{\prime}$ is inconsistent, which contradicts the assumed Robinson property of $L$.

1.4 LEMMA. Under the hypotheses of Theorem 1.2, if $|\mathfrak{A}|<\omega_{n}$ and $\mathfrak{A}=\langle A,<$, $\ldots\rangle \in \operatorname{Str}(\tau)$ is a single-sorted expansion of a well-ordered structure $\langle A,\langle\rangle \simeq$ $\left\langle\lambda,\langle\rangle\right.$ with $\lambda \leqslant \omega_{n}$, then $\bmod _{L}$ th $_{L} \mathfrak{A}=I \mathfrak{X}$.

Proof. Let $\mathfrak{B} \equiv_{L} \mathfrak{A}$; then, by the reduct property of logic $L$ we have that $\mathfrak{B} \uparrow\{<\} \equiv_{L} \mathfrak{A} \uparrow\{<\}$. By definition of $\mathfrak{A}$ and by the assumed characterizability of $\lambda$, we thus have that $\mathfrak{B} \uparrow\{<\} \cong \mathfrak{A} \uparrow\{<\} \cong\langle\lambda,<\rangle$. Therefore, by the isomorphism property of logic $L$, we can safely assume that $\mathfrak{B} \uparrow\{<\}=\mathfrak{A} \uparrow\{<\}$ $=\langle\lambda,<\rangle$.

Now if $\mathfrak{B} \neq \mathfrak{A}$ (absurdum hypothesis) there is some symbol $S \in \tau$ (to fix ideas, let $S$ be a unary relation symbol) and there is some ordinal $\mu<\lambda$ such that

$$
\mathfrak{A} \vDash S[\mu] \text { but } \mathfrak{B} \vDash \neg S[\mu] \quad \text { (or vice versa). }
$$

(In the general case, for $S$ having many places, one shall work with a finite sequence of ordinals smaller than $\lambda$.) Let $T^{\prime}$ be $\operatorname{th}_{L}\langle\mathfrak{A}, \mu\rangle$ in type $\tau \cup\left\{m^{\prime}\right\}$. Let $T^{\prime \prime}$ be $\operatorname{th}_{L}\langle\mathfrak{B}, \mu\rangle$ in type $\tau \cup\left\{\boldsymbol{m}^{\prime \prime}\right\}$ (with $\boldsymbol{m}^{\prime}$ and $\boldsymbol{m}^{\prime \prime}$ new constant symbols). Let $T_{\lambda}$ (resp. $T_{\mu}$ ) characterize $\langle\lambda,\langle\rangle$ (resp. $\langle\mu,<\rangle$ ) in type $\{<\}$ (here, and only here, we are using symbol $<$ instead of $E$ to gain intuition). Let $T_{\mu}^{\prime}$ (resp. $T_{\mu}^{\prime \prime}$ ) say that the order type of the set of predecessors of $m^{\prime}$ (resp. of $m^{\prime \prime}$ ) is $\mu . T_{\mu}^{\prime}$ is obtained by relativizing each sentence of $T_{\mu}$ to $\left\{x \mid x<m^{\prime}\right\}$, i.e. to formula $x<m^{\prime}$; similarly, $T_{\mu}^{\prime \prime}$ is the relativization of $T_{\mu}$ to formula $x<m^{\prime \prime}$. Notice that

$$
T^{\prime} \supseteq T_{\mu}^{\prime} \cup T_{\lambda} \text { and } T^{\prime \prime} \supseteq T_{\mu}^{\prime \prime} \cup T_{\lambda} .
$$

We now claim that $T^{\prime} \cup T^{\prime \prime}$ is inconsistent; as a matter of fact, if $\mathfrak{R} T^{\prime} \cup T^{\prime \prime}$ 
then

$$
\mathfrak{R} \vDash T_{\mu}^{\prime} \cup T_{\mu}^{\prime \prime} \cup T_{\lambda} \cup\left\{S m^{\prime} \wedge \neg S m^{\prime \prime}\right\} .
$$

On the other hand, the assumed properties of $T_{\mu}^{\prime}, T_{\mu}^{\prime \prime}, T_{\lambda}$ ensure that $\mathfrak{N} m^{\prime}=m^{\prime \prime}$, thus $\mathfrak{R} \vDash m^{\prime}=m^{\prime \prime} \wedge S m^{\prime} \wedge \neg S m^{\prime \prime}$ which is impossible.

The lemma is now proved by noting that the inconsistency of $T^{\prime} \cup T^{\prime \prime}$ contradicts the assumed Robinson property.

1.5 Proof of Theorem 1.2. Let $\mathfrak{B} \equiv_{L} \mathfrak{A}$ be single-sorted structures of type $\tau_{0}$; let $|\mathfrak{A}|=\lambda \leqslant \omega_{n}$. By characterizability of $|\mathfrak{A}|$ (Lemma 1.3) and by reduct we have $|\mathfrak{B}|=\lambda$. Expand $\mathfrak{A}$ to $\mathfrak{A}^{\prime}=\langle\mathfrak{A}, E\rangle$ in type $\tau_{1}$ and $\mathfrak{B}$ to $\mathfrak{B}^{\prime}=\langle\mathfrak{B}, F\rangle(E \neq F$ new binary relation symbols) in type $\tau_{2}$, in such a way that

$$
\langle A, E\rangle \cong\langle B, F\rangle \cong\left\langle\lambda,\langle\rangle \leqslant\left\langle\omega_{n},\langle\rangle .\right.\right.
$$

Both th $\mathfrak{A}^{\prime}$ and $\mathrm{th}_{L} \mathfrak{B}^{\prime}$ are consistent extensions of th $\mathfrak{L}_{L} \mathfrak{A}$, resp., in type $\tau_{1}$ and $\tau_{2}$ with $\tau_{1} \cap \tau_{2}=\tau_{0}$. Hence, by the Robinson property there exists $\mathfrak{M} \in \operatorname{Str}\left(\tau_{1} \cup \tau_{2}\right)$ such that

$$
\mathfrak{U}^{\prime} \equiv_{L} \mathfrak{M} \uparrow \tau_{1} \quad \text { and } \quad \mathfrak{B}^{\prime} \equiv_{L} \mathfrak{M} \uparrow \tau_{2}
$$

By Lemma 1.4 noting that $\mathfrak{A}^{\prime}$ is an expansion of a well-ordered structure of order type $\leqslant \omega_{n}$ we have $\mathfrak{A}^{\prime} \cong \mathfrak{M} \uparrow \tau_{1}$. Similarly, we have that $\mathfrak{B}^{\prime} \cong \mathfrak{M} \uparrow \tau_{2}$. By reduct $\mathfrak{U} \cong \mathfrak{M} \uparrow \tau_{0} \cong \mathfrak{B}$. Q.E.D.

1.6 COROllary. If logic $L$ has the Robinson property, $n \in \omega$, and each ordinal $\lambda \leqslant \omega_{n}$ is characterizable by its theory in $\{E\}$ ( $E$ a binary relation symbol), then each ordinal $\mu<\omega_{n+1}$ is characterizable by its theory in $\{E\}$.

Proof. Immediate from Theorem 1.2.

\section{Jumping to $\omega_{n+1}$.}

2.1 TheORem. Assume that logic $L$ has the Robinson property and is not countably compact. Let $n \in \omega$ and assume that each ordinal $\lambda<\omega_{n+1}$ can be characterized by its theory $T_{\lambda}$ in type $\{E\}$ ( $E$ a binary relation symbol). Then $\omega_{n+1}$ itself can be characterized by its theory in type $\{E\}$.

Proof. By [MS, 6.6(iii)], the fact that $L$ is not $(\omega, \omega)$-compact implies that $L$ is not $\omega_{n+1}$-relatively compact, i.e. there are theories

$$
\Sigma=\left\{\varphi_{\alpha} \mid \alpha<\omega_{n+1}\right\}
$$

and $\Gamma$ such that $\Sigma \cup \Gamma$ is inconsistent, but for any subset $\Sigma_{0} \subseteq \Sigma$ with $\left|\Sigma_{0}\right|<\omega_{n+1}$, $\Sigma_{0} \cup \Gamma$ is consistent. Let $\tau$ be the type of $\Sigma \cup \Gamma$. For any ordinal $\beta<\omega_{n+1}$ define $\Sigma_{\beta}=\left\{\varphi_{\alpha} \mid \alpha<\beta\right\}$ and let $\mathfrak{U}_{\beta}$ be a model of $\Sigma_{\beta} \cup \Gamma$. It is no loss of generality to assume that the universes of the $\mathfrak{A}_{\beta}$ are pairwise disjoint and that $\tau$ has only relation symbols. Now expand $\tau$ to $\tau^{\prime}$ by adding a new sort $\sigma$ and new symbols $f, E$, $F, a_{\kappa}$ (for all ordinals $\kappa<\omega_{n+1}$ ) where $E$ and $F$ are binary relations (to be interpreted) over the new sort, the $a_{\kappa}$ 's are constants over $\sigma$, and $f$ is a unary function taking elements of the old sorts of $\tau$ into elements of $\sigma$. Define $\mathfrak{M} \in$ $\operatorname{Str}\left(\tau^{\prime}\right)$ as follows (here, for simplicity, symbols are identified with their interpretation): 


$$
\begin{gathered}
\mathfrak{M} \uparrow\left\{E, a_{\kappa}\right\}_{\kappa<\omega_{n+1}}=\left\langle\omega_{n+1},<, \kappa\right\rangle_{\kappa<\omega_{n+1}} ; \\
\quad \text { range of } f=\omega_{n+1} ; \\
\text { domain of } f=\left(\text { union of the universes of the } \mathfrak{A}_{\beta}\right)_{\beta<\omega_{n+1}} ; \\
f^{-1}(\kappa)=\text { universe of } \mathfrak{A}_{\kappa} \quad\left(\kappa<\omega_{n+1}\right) ; \\
(\mathfrak{M} \uparrow \tau) \mid f^{-1}(\kappa)=\mathfrak{A}_{\kappa} \quad\left(\kappa<\omega_{n+1}\right), \text { i.e. the substructure of } \mathfrak{M} \uparrow \tau \\
\text { generated by the inverse image } f^{-1}(\kappa) \text { is just } \mathfrak{A}_{\kappa} ; \\
F \lambda \mu \text { holds in } \mathfrak{M} \text { iff } \lambda, \mu<\omega_{n+1} \text { and } \mathfrak{A}_{\lambda} \vDash \varphi_{\mu} .
\end{gathered}
$$

Roughly, $\mathfrak{M}$ is a disjoint union of the $\mathfrak{A}_{\beta}$ together with a "satisfaction" predicate $F$ relating $\mathfrak{A}_{\beta}$ to the sentences of $\Sigma$. Structure $\mathfrak{M}$ satisfies theory $T$ of type $\tau^{\prime}$ given by the following sentences (here we use the small Greek letters $\zeta, \eta, \theta$, for variables on the new sort $\sigma$ ).

$(A \times 1)_{\kappa<\lambda<\omega_{n+1}} . E$ is a linear ordering of the elements of the new sort $\sigma$ and $E a_{\kappa} a_{\lambda}$. $(\mathrm{Ax} 2)_{\kappa<\omega_{n+1}}$. The order type of the set of predecessors of $a_{\kappa}$ is $\langle\kappa,\langle\rangle$ (one has just to relativize to $\left\{x \mid E x a_{\kappa}\right\}$ theory $T_{\kappa}$ which is assumed, by hypothesis, to characterize $\langle\kappa,<\rangle)$.

$(\mathrm{Ax} 3)_{s \in \text { sorts of } \tau} \cdot \forall \zeta \exists x^{s} f\left(x^{s}\right)=\zeta$ (i.e. whenever $\zeta$ is of sort $\sigma$ its inverse image under $f$ is nonnull on each sort of type $\tau$ ).

$(\mathrm{Ax4})_{\beta<\omega_{n+1}} \forall \eta\left(F \eta a_{\beta} \rightarrow \varphi_{\beta}^{(x \mid f(x)=\eta\})}\right.$ (i.e. in the $\eta$ th universe $\varphi_{\beta}$ is satisfied if $\left.F \eta a_{\beta}\right)$.

(Ax5). $\forall \zeta \forall \eta E \eta \zeta \rightarrow F \zeta \eta$ (so that, by (Ax4) theory $\Sigma_{\zeta}$ is satisfied in the $\zeta$ th universe).

(Ax6) ${ }_{\gamma \in \Gamma^{*}} \forall \zeta \gamma^{\{x \mid f(x)-\zeta\}}$ (so that $\Gamma$ is satisfied in the $\zeta$ th universe).

Claim 1. $\forall \mathfrak{N} \vDash T$ there is no $m \in N(N$ the universe of $\mathfrak{R})$ such that $\mathfrak{R} \vDash E a_{\kappa}[m]$ for all $\kappa<\omega_{n+1}$. As a matter of fact, assume such $\mathfrak{N}$ and $m$ exist (absurdum hypothesis). Let $\mathfrak{N}^{\prime}=\langle\mathfrak{N}, m\rangle$; then in $\mathfrak{N}^{\prime}$ the following hold:

$$
\begin{gathered}
f^{-1}(m) \neq \varnothing \text { on each sort of } \tau \quad(\text { by }(\mathrm{Ax} 3)), \\
\Gamma \text { holds when relativized to } f^{-1}(m) \quad(\text { by }(\mathrm{Ax} 6)), \\
\left(F m a_{\kappa}\right)_{\kappa<\omega_{n+1}} \text { by }(\mathrm{Ax} 5) \text { and assumption about } m, \\
\left(\varphi_{\kappa}^{\{x \mid f(x)=m\}}\right)_{\kappa<\omega_{n+1}} \text { by }(\mathrm{Ax} 4),
\end{gathered}
$$

i.e. $\Sigma$ holds when relativized to $f^{-1}(m)$. Therefore in $f^{-1}(m)$ theory $\Sigma \cup \Gamma$ holds, a contradiction.

Claim 2. $\forall \mathfrak{N} \vDash T, \mathfrak{R} \uparrow\{E\} \cong\left\langle\omega_{n+1},<\right\rangle$. As a matter of fact, since $\left.|\mathfrak{N} \uparrow\{E\}|\right\rangle$ $\omega_{n+1}$ by (Ax1), it suffices to show that $\Re$ is well ordered and each $m \in N$ has at most $\omega_{n}$ many predecessors. Assume $\mathfrak{R} \vDash T$ and $\mathfrak{N}$ is not well ordered (absurdum hypothesis). Then $E$ is a linear ordering having some infinitely descending chain $c_{0}>c_{1}>\cdots$, but $c_{0}$ cannot bound the $a_{\kappa}$ by Claim 1, so by letting $a_{\lambda}$ (for some $\left.\lambda<\omega_{n+1}\right)$ be greater than $c_{0}$ we have that the set of predecessors of $a_{\lambda}$ is not well ordered, thus contradicting (Ax2), in view of the characterizability of $\langle\lambda,\langle\rangle$ by $T_{\lambda}$. 
Similarly, if $\mathfrak{N}$ has some element $m \in N$ with $\mu$ many predecessors, $\mu$ a cardinal $>\omega_{n}$, then by Claim 1 there exists $\lambda<\omega_{n+1}$ such that $a_{\lambda}$ is greater than $m$; hence $a_{\lambda}$ cannot satisfy (Ax2), a contradiction. This proves Claim 2 .

Claim 3. (END of THE PRoOf OF Theorem 2.1.) Let $T_{0}=\mathrm{th}_{L}\left\langle\omega_{n+1},\langle\rangle\right.$ in type $\{E\}$ ( $E$ a binary relation symbol). Then

$$
\forall \mathfrak{N} \vDash T_{0}, \mathfrak{N} \cong\left\langle\omega_{n+1},\langle\rangle .\right.
$$

As a matter of fact, assume $\mathfrak{N} \vDash T_{0}$ with $\mathfrak{N} \approx\left\langle\omega_{n+1},\langle\rangle\right.$ (absurdum hypothesis). Then

(i) either $\mathfrak{R}$ is not well ordered,

(ii) or some $m \in N$ has more than $\omega_{n}$ predecessors,

(iii) or, finally, $|\mathfrak{N}|<\omega_{n+1}$.

(i) If $\mathfrak{R}$ is not well ordered then there is some infinitely descending chain $c_{0}>c_{1}>\cdots$, so there is an expansion $\mathfrak{N}^{\prime}=\left\langle\mathfrak{R}, c_{r}\right\rangle_{r \in \omega}$ satisfying theory $T^{\prime}$ defined by $T^{\prime}=T_{0} \cup\left\{E c_{r+1} c_{r} \mid(r \in \omega)\right\}$. On the other hand, by Claim 2, the $E$-reduct of any model of $T$ is well ordered, so that $T^{\prime} \cup T \cup T_{0}$ is inconsistent; but this contradicts the assumed Robinson property of $L$ (notice that $T_{0}$ is complete in $\left.\{E\}=\operatorname{type}\left(T^{\prime}\right) \cap \operatorname{type}(T)\right)$.

(ii) If $m \in N$ has more than $\omega_{n}$ many predecessors, say $\left\{c_{k}\right\}_{\kappa<\omega_{n+1}}$, consider the expansion $\mathfrak{N}^{\prime}=\left\langle\mathfrak{N}, m, c_{\kappa}\right\rangle_{\kappa<\omega_{n+1}}$ and let $T^{\prime \prime}$ be the complete theory of $\mathfrak{N}^{\prime}$ in type $\tau^{\prime \prime}=\left\{E, m, c_{\kappa}\right\}_{\kappa<\omega_{n+1}}$. By arguing as in (i) above we see that $T^{\prime \prime} \cup T \cup T_{0}$ is inconsistent; but this contradicts the assumed Robinson property.

(iii) Assume finally $|\mathfrak{N}|=\omega_{m}<\omega_{n+1}$. Expand $\mathfrak{R}$ to $\mathfrak{N}^{\prime}=\left\langle\mathfrak{N}, E^{\prime}\right\rangle\left(E^{\prime}\right.$ a new binary relation symbol) by stipulating that $\mathfrak{R}^{\prime}\left\{\left\{E^{\prime}\right\} \cong\left\langle\omega_{m},<\right\rangle\right.$. Since by assumption $\left\langle\omega_{m},\langle\rangle\right.$ can be characterized by its complete theory $T_{\omega_{m}}$ in type $\left\{E^{\prime}\right\}$, we have that all the models of theory $T_{0} \cup T_{\omega_{m}}$ have cardinality equal to $\omega_{m}$. Hence $T_{0} \cup T \cup T_{\omega_{m}}$ is inconsistent, again contradicting the assumed Robinson property. This proves Theorem 2.1. Q.E.D.

\section{Categoricity and compactness.}

3.1 TheORem. Let logic $L$ have the Robinson property. Assume that $L$ is not $(\omega, \omega)$-compact. Then for any single-sorted structure $\mathfrak{A}$ with $|\mathfrak{X}|<\omega_{\omega}$ we have

$$
\bmod _{L} \operatorname{th}_{L} \mathfrak{A}=I \mathfrak{A} \text {. }
$$

Proof. Let $|\mathfrak{A}|=\omega_{n}$; the proof is by induction on $n$.

The case $n=0$. By Proposition $1.1\langle\omega,\langle\rangle$ is characterizable by its complete theory; then by Theorem 1.2 each single-sorted countable structure is characterizable by its theory.

The case $n+1$. By the induction hypothesis Theorem 3.1 holds for $n$, i.e. each single-sorted structure of cardinality $\leqslant \omega_{n}$ is characterizable. In particular, each ordinal $\lambda<\omega_{n+1}$ is characterizable. By Theorem $2.1 \omega_{n+1}$ itself is characterizable, hence each ordinal $\mu \leqslant \omega_{n+1}$ is characterizable. Then, again by Theorem 1.2, each single-sorted structure $\mathfrak{A}$ with $|\mathfrak{A}| \leqslant \omega_{n+1}$ is characterizable. Q.E.D.

3.2 Remarks. (i) The single-sortedness hypothesis is just for simplicity.

(ii) Theorem 3.1 roughly states that any logic having the Robinson property is 
either countably compact or has a very strong expressive power (in fact the strongest possible up to cardinal $\left.\omega_{\omega}\right)$.

(iii) A number of consequences can be drawn from Theorem 3.1 by either limiting the size of $L$ or by making purely set-theoretical assumptions.

The following corollary links the Robinson consistency with the Craig interpolation theorem; it improves [MS, 6.11] where the same result is obtained with the help of an additional "Feferman-Vaught" hypothesis. For the definition of $L_{\omega \omega}\left(Q^{i}\right)_{i \in I}$ see [MSS].

3.3 Corollary. Assume that $2^{\omega}<2^{\omega_{n}}$ for some $n \in \omega$. Let $L=L_{\omega \omega}\left(Q^{i}\right)_{i \in \omega}$ have the Robinson property; then in $L$ the Craig interpolation theorem holds and $L$ is $(\omega, \omega)$-compact.

Proof. We first claim that $L$ is $(\omega, \omega)$-compact. If not, then $\forall n \in \omega$ and $\forall s \subseteq \omega_{n}$ the complete theory of $\left\langle\omega_{n},\langle, s\rangle\right.$ in type $\{E, S\}$ ( $E$ binary, $S$ unary relation symbols) can characterize $\left\langle\omega_{n},\langle, s\rangle\right.$ up to isomorphism, by Theorem 3.1. Thus

number of theories of $L$ of type $\{E, S\}$

$\geqslant$ number of isomorphism classes $I\left\langle\omega_{n},\langle, s\rangle\right.$ for $s \subseteq \omega_{n}$, i.e. $2^{\omega_{n}}$.

Now notice that in any countably generated logic, such as $L$, the number of theories of finite type cannot exceed $2^{\omega}$. Thus $2^{\omega}>2^{\omega_{n}} \forall n \in \omega$ contradicting our set-theoretical assumption.

Therefore $L$ is $(\omega, \omega)$-compact; but the Robinson property and countable compactness for a countably generated logic are well known to imply the Craig interpolation theorem (see [MS, 2.1]). Q.E.D.

4. The case $L \geqslant L\left(Q_{1}\right)$. Let $L\left(Q_{1}\right)$ be the logic with quantifier "there exist uncountably many".

4.1 THEOREM. If $L \geqslant L\left(Q_{1}\right)$ satisfies the Robinson consistency theorem, then

(i) $L$ is not axiomatizable, nor countably compact,

(ii) $\forall \mathfrak{U}$ with $\mathfrak{A}$ single-sorted and $|\mathfrak{A}|<\omega_{\omega}$, we have

$$
\bmod _{L} \operatorname{th}_{L} \mathfrak{A}=I \mathfrak{A},
$$

(iii) if $E$ is a binary relation symbol and $S$ is unary, then

$$
2^{\left|\mathbf{S t c}_{L}\{E, S\}\right|} \geqslant 2^{\omega_{n}} \text { for any } n \in \omega .
$$

Proof. (i) has been proved in [Mu1]. (ii) follows immediately from (i) in the light of Theorem 3.1. (iii) Since $L$ is not countably compact by (i), then we can argue as in the proof of Corollary 3.3 to conclude that

number of theories of $L$ of type $\{E, S\}=2^{\left|\operatorname{Stc}_{L}\{E, S\}\right|} \geqslant 2^{\omega_{n}}$ for all $n \in \omega$.

4.2 Corollary. Assume that $2^{\omega}<2^{\omega_{n}}$ for some $n \in \omega$; then no countably generated (i.e. having countably many quantifiers) extension $L$ of $L\left(Q_{1}\right)$ satisfies the Robinson consistency theorem.

Proof. Assume that $L$ is a counterexample; then by Theorem 4.1(iii) we have that $2^{\omega} \geqslant 2^{\omega_{n}} \forall n \in \omega$, since $\left|\operatorname{Stc}_{L}\{E, S\}\right|=\omega$ in any countably generated logic $L$. 
This contradicts our set-theoretical assumption.

Something weaker than $\mathrm{CH}$ also yields

4.3 Corollary. Assume that $\omega_{n}=2^{\omega}$ for some $n \in \omega$; then if $L \geqslant L\left(Q_{1}\right)$ satisfies the Robinson consistency theorem, the system of real numbers is characterizable by its complete theory in $L$.

Proof. Then there are $\omega_{n}$-many reals; now apply Theorem 4.1(ii).

5. An identity. Let $\neg L^{\mu}$ abbreviate the following set-theoretical statement:

"there is no inner model with a measurable cardinal $>\omega$ ".

Recall that compactness means $(\kappa, \omega)$-compactness for all $\kappa \geqslant \omega$.

5.1 TheOREM (Assuming $\neg 0^{\#}$, OR EVEN $\neg L^{\mu}$ ). In logic $L$, let $\operatorname{Stc}_{L}(\tau)$ be a set whenever $\tau$ is a set; then $L$ satisfies the Robinson consistency theorem iff $L$ is compact and satisfies the Craig interpolation theorem.

Proof. In [Mu3] the theorem is proved under $\neg 0^{\sharp}$; but Claim 2 thereof (which is the only point where $\neg 0^{\#}$ is used) is actually a consequence of [MS, 6.6(iv)] only. The latter, in turn, only depends on [MS, 6.4(ii)] and on the fact that for any infinite regular cardinal $\kappa$, for any uniform ultrafilter $D$ on $\kappa, D$ is $\lambda$-descendingly incomplete for all infinite $\lambda$. Now this final statement is well known to be weaker than $\neg L^{\mu}$; see [MS, 6.5(iii)] or [DJK].

5.2 Remark. For logics $L$ in which $\operatorname{Stc}_{L}(\tau)$ is a set if so is $\tau, \neg L^{\mu}$ also yields that "compactness = JEP", the latter being the Joint Embedding Property for $L$-elementary embeddings. Use the argument found in [Mu4] where one also can find a complete proof of Theorem 5.1. The same identities have been independently proved in [MS1] by assuming that there is no measurable cardinal $>\omega$ and that $L$ has an "occurrence number".

6. H. Friedman's problems. H. Friedman's third problem in [Fr] of finding logics strictly between $L_{\infty \omega}$ and $L_{\infty \infty}$ satisfying the Craig interpolation theorem is still unsolved; but (a refinement of) the lemmas in $\$ \S 1$ and 2 of this paper (see [Mu5]) yield the following.

6.1 TheOREM (Assuming $\neg 0^{\#}$ OR $\neg L^{\mu}$ ). No logic $L$ between $L_{\infty}$ and $L_{\infty}$ satisfies the Craig interpolation theorem, nor the Robinson consistency theorem, unless $\equiv_{L}=\cong$.

Proof. In [Mu5, Theorems 5.5 and 5.7] the theorem is proved under assumption $\neg 0^{\sharp}$; but $\neg 0^{\sharp}$ is only needed for the proof of Theorem 5.4 therein. The latter theorem, however, still holds under assumption $\neg L^{\mu}$, by the observation made in the proof of Theorem 5.1.

Also H. Friedman's fourth problem, asking for proper extensions of first order logic which satisfy compactness and interpolation, is still unsolved. Notice that one might equivalently ask for extensions satisfying compactness and the Robinson consistency theorem, or even, in the light of Theorem 5.1 (under some suitable hypotheses), ask for extensions satisfying the Robinson consistency theorem alone. 
6.2 TheOREM. Let $L$ be a countably-generated logic satisfying the Robinson consistency theorem. Assume that every consistent theory $T$ with $|T| \leqslant \omega$ has a model of cardinality $\leqslant \omega$. Then $L \sim L_{\omega \omega}$.

Proof. $L$ must be countably compact, for otherwise, by Theorem 3.1, th ${ }_{L}\left\langle\omega_{1},\langle\rangle\right.$ would be a consistent countable theory of type $\{<\}$ having no countable model, thus contradicting our (Löwenheim) assumption. Besides countable compactness, $L$ also has its Löwenheim number (for sentences) equal to $\omega$. Now apply the Lindstrom theorem (see [Li] or [Fl]). Q.E.D.

To prove Theorem 6.3 we need to relativize the Robinson property to a class of structures. More precisely, let $X$ be a class of structures; then, following [Mu2], we say that logic $L$ satisfies the Robinson consistency theorem on $X$ iff

$\forall \mathfrak{M} \in X \cap \operatorname{Str}\left(\tau^{\prime}\right), \forall \mathfrak{R} \in X \cap \operatorname{Str}\left(\tau^{\prime \prime}\right)$, if $\tau=\tau^{\prime} \cap \tau^{\prime \prime}$ and $\mathfrak{M} \uparrow \tau \equiv_{L} \mathfrak{N} \uparrow \tau$,

then $\exists \mathfrak{A} \in X \cap \operatorname{Str}\left(\tau^{\prime} \cup \tau^{\prime \prime}\right)$ with $\mathfrak{A} \uparrow \tau^{\prime} \equiv_{L} \mathfrak{M}$ and $\mathfrak{A} \uparrow \tau^{\prime \prime} \equiv_{L} \mathfrak{R}$.

This definition is a natural generalization of Definition 0.2 ; when $X$ is the class of all structures then clearly $L$ satisfies the Robinson consistency theorem on $X$ iff $L$ satisfies the Robinson consistency theorem in the sense of Definition 0.2. Let $K$ be the class of all countable structures of finite type, and $Y$ be the class of all structures; we can prove the following result.

6.3 TheOREM $\left(\neg 0^{\#}\right.$ OR $\left.\neg L^{\mu}\right)$. Let $L=L_{\omega \omega}\left(Q^{i}\right)_{i \in I}$ (with I a set) satisfy the Robinson consistency theorem on $Y$ as well as on $K$. Then $\mathfrak{A} \equiv_{L} \mathfrak{B}$ iff $\mathfrak{A} \equiv \mathfrak{B}$, for any two countable structures $\mathfrak{A}$ and $\mathfrak{B}$.

Proof. By the main theorem in [Mu2], $\equiv_{L}$ on $K$ is either equal to $\equiv$ or to $\cong$. By Theorem 5.1, $L$ is compact; thus it suffices to prove our theorem only for $\mathfrak{A}$ and $\mathfrak{B}$ having finite type, since each sentence of $L$ will only depend on a finite number of symbols. Assume $\equiv_{L}=\cong$ (absurdum hypothesis). Let $T$ be any first order theory which is consistent and complete in the finite type $\tau$, having one sort $s$, and which is $\omega_{1}$-categorical but not $\omega$-categorical; then $T$ has exactly $\omega$ many nonisomorphic countable models (Baldwin and Lachlan, [BL]), $\mathfrak{N}_{0}, \mathfrak{N}_{1}, \ldots, \mathfrak{N}_{n}, \ldots$, ( $n \in \omega$ ). On the other hand, $\forall n \in \omega$, th $_{L} \mathfrak{N}_{n}$ is $\omega$-categorical by the absurdum hypothesis, and has models of arbitrarily large cardinality, by compactness. Let $C_{n}$ be the class of cardinals $\kappa$ such that $\mathrm{th}_{L} \mathfrak{N}_{n}$ has a model of cardinality $\kappa$; then $C_{n} \cap C_{m}=\{\omega\}$ by the Morley theorem applied to $T$. Let $\rho: \tau \rightarrow \tau^{\rho}$ be a namechanger with $\tau \cap \tau^{\rho}=\{s\} ; \rho$ naturally transforms $\mathfrak{R}_{0}$ into $\mathfrak{R}_{0}^{\rho} \in \operatorname{Str}\left(\tau^{\rho}\right)$; then, th $\mathfrak{N}_{0}^{\rho} \cup$ th $\mathfrak{R}_{1}$ has only countable models, as $C_{0}$ is disjoint from $C_{1} \backslash\{\omega\}$, which contradicts the fact that $L$ is compact. Q.E.D.

\section{REFERENCES}

[Ba] K. J. Barwise, Axioms for abstract model theory, Ann. Math. Logic 7 (1974), 221-265.

[BL] J. T. Baldwin and A. H. Lachlan, On strongly minimal sets, J. Symbolic Logic 36 (1971), 79-96.

[Br] K. B. Bruce, Ideal models and not so ideal problems in the model theory of $L(Q)$, J. Symbolic Logic 43 (1978), 304-321.

[DJK] T. Dodd, R. B. Jensen and B. J. Koppelberg, Some applications up to $K$, Lecture Notes in Math. (to appear). 
[Fe1] S. Feferman, Applications of many-sorted interpolation theorems, Proc.Sympos. Pure Math., Vol. 25, Amer. Math. Soc., Providence, R. I., 1974, pp. 205-223.

[Fe2] , Two notes on abstract model theory. I, Fund. Math. 82 (1974), 153-165; II, 89 (1975), 111-130.

[FI] J. Flum, First order logic and its extensions, Lecture Notes in Math., Vol. 499, Springer-Verlag, Berlin and New York, 1975, pp. 248-310.

[Fr] H. Friedman, One hundred and two problems in mathematical logic, J. Symbolic Logic 40 (1975), 113-129.

[Hu] J. Hutchinson, Model theory via set theory, Israel J. Math. 24 (1976), 286-304.

[Li] P. Lindstrom, On extensions of elementary logic, Theoria 35 (1969), 1-11.

[MS] J. A. Makowsky and S. Shelah, The theorems of Beth and Craig in abstract model theory. I. The abstract setting, Trans. Amer. Math. Soc. 256 (1979), 215-239.

[MS1] __ Positive results in abstract model theory, Ann. Math. Logic (to appear).

[MSS] J. A. Makowsky, S. Shelah and J. Stavi, $\Delta$-logic and generalized quantifiers, Ann. Math. Logic 10 (1976), 155-192.

[Mu1] D. Mundici, Applications of many-sorted Robinson consistency theorem, Z. Math. Logik 27.1 (to appear).

[Mu2] _ An algebraic result about soft model theoretical equivalence relations with an application to H. Friedman's fourth problem, J. Symbolic Logic (to appear).

[Mu3] _ Compactness + Craig interpolation = Robinson's consistency in any logic (preprint, 1979).

[Mu4] _ Compactness = JEP in any logic, Fund. Math. (to appear).

[Mu5] _ Compactness, interpolation and H. Friedman's third problem (to appear).

Centro Nazionale delle Ricerche, C. N. R., Loc. Romola N.76, 50060 donnini (Florence), ITALY 